\title{
Digital collection of morphological variability of wheat spike
}

\author{
M. Genaev ${ }^{1 *}$, E. Komyshev ${ }^{1}$, F. Hao ${ }^{2,3}$, V. Koval ${ }^{1}$, N. Goncharov ${ }^{1,4}$, D. Afonnikov ${ }^{1,2}$ \\ ${ }^{1}$ Institute of Cytology and Genetics SB RAS, Novosibirsk, Russia \\ ${ }^{2}$ Novosibirsk State University, Novosibirsk, Russia \\ ${ }^{3}$ Heilongjiang University, Harbin, China \\ ${ }^{4}$ Novosibirsk State Agrarian University, Novosibirsk, Russia \\ *e-mail:mag@bionet.nsc.ru
}

Key words: wheat, spike characteristics, phenotyping, annotation, data integration

Motivation and Aim: The structure of the ear is one of the most important features of cereals associated with such agronomically important traits as productivity, resistance to environmental factors and pests, threshebility. Ears differ in shape, size, density, ostectomy, color, etc. Analysis of the ear traits requires visual inspection, manual measurements and is very time-consuming.

Methods and Algorithms: The effectiveness of ears' phenotyping can be improved by the introduction of an automated image processing technology, storage of information in databases, use of machine learning algorithms to analyze this information. In this work, we present a new approach for collecting, storing and analyzing of information about morphometric characteristics of ears of wheat. Two protocols for obtaining digital images of the ear have been developed.

Results: The computer-aided information system SpikeDroidDB has been developed, which allows you to store digital images of the ear, annotate their phenotypic features (14 features, including plant variety description, links to parent genotypes, generation, plant sowing number, ear morphology description). The interface provides a flexible query system to access the data. SpikeDroidDB represents an interconnected representation between genotype, phenotype, location, and growing conditions. The web interface of SpikeDroidDB is available at http://spikedroid.biores.cytogen.ru and allows you to work with the system as with desktop computers or mobile devices. We used SpikeDroidDB for the digitization and annotation of a collection of ears of F2 hybrids from crosses between the Australian cultivar of common wheat Triple Dirk and accession KU506 of Chinese wheat Triticum yunnanense. This experiment includes analysis of 103 plants, 230 spike images.

Conclusion: The analysis of the variability of ears in form, length, and other traits allowed determination of the type of their genetic control: compactness is controlled by two recessive genes, awn type and hairiness at the site of attachment of the spikelet to the axis is controlled by single dominant gene type, hairiness on the axis of the spike is controlled by two dominant genes.

Acknowledgements: The work supported by the RSF grant No. 17-74-10148. 